\title{
CONDICIONES DE BASE PARA LA REINTEGRACIÓN SOCIAL EN EL POSCONFLICTO ${ }^{1}$ Basic conditions for social reintegration in the post-conflict
}

\section{John Jairo García Peña}

Docente e investigador escuela de posgrados, Universidad Católica Luis Amigó. john.garciape@amigo.edu.co ORCID: 0000-0001-8390-5945

\section{Claudia Marcela Arana Medina}

Docente Universidad de Sanbuenaventura y Universidad Católica Luis Amigó, claudia.araname@amigo.edu.co ORCID: 0000-0002-3660-9526

Recibido: 21/10/2018 • Aprobado: 15/11/2018

Cómo citar: García Peña, J., \& Arana Medina, C. (2018). Condiciones de base para la reintegración social en el posconflicto. Ciencia y Sociedad, 43(4), 69-81. doi: http://dx.doi.org/10.22206/cys.2018.v43i4.pp69-81

\section{Resumen}

Este artículo surge de una investigación, cuyo objetivo fue aportar a las condiciones psicosociales de base, de las personas que deciden desvincularse del conflicto armado colombiano, teniendo presente el contexto en el que han estado inmersas y al que se reintegrarán. El estudio asumió un diseño cualitativo, enmarcado desde un estudio teórico, cuya revisión se hizo en revistas y páginas Web de acceso abierto. Los resultados expresan la importancia de fortalecer con esta población el concepto de ciudadanía desde la perspectiva de Derecho, dentro del reconocimiento de categorías como bienestar psicológico, desarrollo humano y social, que apuesten por la construcción de ciudadanos con derechos en el ejercicio pleno de sus facultades políticas y socioculturales, a partir del reconocimiento de su dignidad, como partícipes de un territorio. Como conclusión relevante, estos conceptos se presentan como un eje conductor, que hará posible, que en un país en posconflicto, este proceso se asuma con responsabilidad y éxito, reconociendo la función relevante que cumple la sociedad civil para que un Estado salga del circuito de la guerra.

Palabras clave: Posconflicto; Reintegración social; Bienestar psicológico; Desarrollo Humano; Derechos Humanos; Ciudadanía.

\section{Abstract}

This article arises from an investigation, whose objective was to contribute to the basic psychosocial conditions of the people who decide to disassociate themselves from the Colombian armed conflict, bearing in mind the context in which they have been immersed and to which they will be reintegrated. The study assumed a design, framed from a theoretical study, which was revised in journals and open access Web pages. The results express the importance of strengthening with this population the concept of citizenship from the perspective of Human Rights, within the recognition of categories such as psychological well-being, human and social development, that bet on the construction of citizens with rights in the full exercise of their political and sociocultural faculties, from the recognition of their dignity, as participants in a territory. As a relevant conclusion, these concepts are presented as a driving axis, which will make possible for a country in a post-conflict process to assume responsibility and success, recognizing the relevant role played by civil society, so that a State leaves the circuit from the war.

Keywords: Post-conflict; Social reintegration; Psychological wellness; Human Development; Human Rights; Citizenship.

1. Derivado de la investigación "Evaluación de la eficacia de un protocolo de diagnóstico e intervención neurocognitivo y psicosocial en personas desvinculadas del conflicto armado en Colombia”, realizada mediante convenio entre la Universidad Católica Luis Amigó y la Universidad de Sanbuenaventura, en Medellín - Colombia, 2017. 


\section{Introducción}

Este artículo pretende acercarse a las condiciones psicosociales de las personas que en el marco del conflicto armado en Colombia han dejado las armas. Es una reflexión, producto de una investigación cuyo objetivo es aportar a los aspectos psicosociales de las personas que deciden desvincularse del conflicto armado colombiano, teniendo presente el contexto psicológico y social en el que han estado inmersos y al que se reintegrarán, como lo son el desarrollo de estrategias que se encuentren encaminadas al fortalecimiento de actitudes para la Paz, enmarcado en el posconflicto, en la ciudad de Medellín, Colombia.

En este contexto y a manera de histórico temático, es de destacar que desde hace varias décadas existe un interés permanente en el mundo por asumir mediante convenios de paz, la solución de los conflictos internos en muchos países. Colombia muy particularmente con un conflicto armado complejo y estructural, ha formulado en su historia moderna, diversos procesos de paz, que han contribuido a su transformación, en la medida que han permitido poner en la agenda pública, asuntos como el restablecimiento de los derechos fundamentales y la promoción del desarrollo humano y social de quienes han participado del conflicto.

En Colombia, desde 1982, se iniciaron conversaciones de paz con la guerrilla, a partir de lo cual, pueden identificarse, entre 1989 y 2003, nueve procesos de paz, que han permitido la desmovilización colectiva de personas que han estado en el conflicto armado, sin incluir el de 2016 con las FARC-EP (Fuerzas armadas revolucionarias de Colombia, ejército del pueblo); entre ellos: los acuerdos con el Movimiento 19 de Abril (M-19), el 2 de enero de 1989, el Partido Revolucionario de los Trabajadores (PRT) el 25 de enero 1991, el Ejército Popular de Liberación (EPL) el 15 de febrero de 1991, el Movimiento Armado Quintín
Lame el 27 de mayo de 1991, el Comando Ernesto Rojas, en abril de 1992, la Corriente de Renovación Socialista (CRS) el 9 de abril de 1994, las Milicias Populares del Pueblo, y para el Pueblo el 26 de mayo de 1994, el Frente Francisco Garnica el 30 de junio de 1994 y las Autodefensas Unidas de Colombia (AUC - Paramilitares) el 15 de julio de 2003 (GMH, 2013).

Mediante estos nueve acuerdos, cerca de 43.000 excombatientes entregaron sus armas en forma colectiva e individual y se reinsertaron a la vida civil, sin embargo, las pocas garantías para generar un proceso adecuado de reintegración, como el laboral, educativo, accesos a la salud y la vivienda, el hacer parte y disfrutar del crédito financiero, etc., llevaron a que en estos procesos hubieran retrocesos que conllevaron a que muchas de estas personas antes en el conflicto armado, reincidieran en un maniobrar ilegal (Centro Nacional de Memoria Histórica - CNMH, 2015).

En abril de 2017, sumando el proceso de paz que se desarrolló con las FARC - EP, la Agencia Colombiana de Reintegración de personas y grupos alzados en armas (2017) reportó en Colombia, 50.227 desmovilizados que ingresaron al programa de reintegración, de los cuales, 28.063 pertenecen a desmovilización colectiva y 22.164 , a la individual. De esta población, el 86,71\% (43.554) son del sexo masculino y el 13,28\% (6.673) corresponde al sexo femenino; sobre el componente ocupacional, muestra que las personas que han culminado su proceso de reintegración (un total de 16.747), sólo el 26,58\% (4.453 personas) se encuentran ocupadas en el sector formal y el 53,048\% (8.884) se encuentran ocupadas en el sector informal.

Otro dato relevante de la Agencia Colombiana para la Reintegración (ACR, 2016), es que de los cerca de 32.000 desmovilizados de las AUC, hasta esa fecha, solo 1.773 , lograron vincularse laboralmente; esto muestra que las políticas de reintegración social carecen de resultados óptimos e inclusive, pueden 
volverse un proceso que incuba fenómenos como las Bandas Criminales - BACRIM o el surgimiento de nuevos accionares delincuenciales.

Colombia, como otros países que han vivido estos procesos, presenta déficit de competencias estatales, para asumir la implementación de los acuerdos de paz, las cuales redundan en la desconfianza de la sociedad civil generada por la poca honestidad, acciones fallidas y compromisos olvidados por las partes negociadoras, pocos beneficios para las víctimas, mecanismos jurídicos difusos para la implementación legítima de lo acordado, la corrupción que desfalca los recursos económicos de la ciudadanía y limita la generación de inversión social, la escasa voluntad política que obstaculiza la fluidez de los procesos de manera planificada y concertada, el escepticismo del sector empresarial que muestra poco compromiso para generar procesos de reintegración equitativos, la apatía de algunos sectores de los grupos armados ilegales para dejar las armas y cambiar de estilo de vida, llevando como consecuencia al nacimiento de bandas emergentes; lo que conlleva a cuestionarse por la viabilidad de alcanzar un verdadero período de posconflicto que logre una paz participativa, estable y duradera.

Una iniciativa de país para afrontar estos procesos sociopolíticos complejos, creando la Agencia Colombiana para la Reintegración - ACR lo constituyen el Decreto 4138 del 03 de noviembre de 2011 y el decreto resultante de su modificación, Decreto 2253 del 4 de noviembre de 2015. La Agencia Colombiana para la Reintegración (ACR) es una unidad delegada para atender los procesos de reintegración de excombatientes, que reglamenta las condiciones y obligaciones para el acceso y otorgamiento de beneficios sociales y económicos de los procesos de reintegración a la sociedad civil de la población desmovilizada.

La ACR, a partir de mayo de 2017, se transforma en ARN, agencia para la reincorporación y normalización, robusteciendo la institucionalidad en el proceso que se adelanta con las FARC-EP, mediante decreto ley 897/2017, que mantiene en vigencia la Resolución 1356 de 2016, en su Art. 3, que autoriza a este organismo estatal, para dirigir y definir la ruta de reintegración, pensada como el conjunto de condiciones, beneficios, estrategias, metodologías y acciones para las personas y grupos alzados en armas, concertados con la persona en proceso de reintegración, para promover el desarrollo de capacidades, la superación de la situación de vulnerabilidad y el ejercicio autónomo de la ciudadanía, lo que se conoce como los procesos DDR - Desarme, Desmovilización y Reintegración (Agencia para la Reincorporación y la Normalización - ARN, 2017).

En términos de antecedentes, Vicenç Fisas (2010), titular de la Cátedra UNESCO sobre Paz y Derechos Humanos de la Universitat Autónoma de Barcelona (UAB) y director de la Escola de Cultura de Pau (ECP), presenta una síntesis de los procesos de paz acaecidos a finales del S. xx e inicios de éste, en once países: Angola, Sudáfrica, Tayikistán, Sierra Leona, sur de Sudán, Burundi, Indonesia (Aceh), Nepal, Guatemala, Nicaragua, El Salvador e Irlanda del Norte. En la misma línea este autor (2011), fundamenta lo que es un programa de Desarme, Desmovilización y Reintegración DDR, con base en los lineamientos de Naciones Unidas, sus objetivos, fases, cronogramas y contextos de su ejecución. Esboza los tipos de desarme y desmovilización que pueden presentarse en un proceso de paz, las diversas tipologías de beneficiarios y el marco legal que favorece un DDR.

A 2011, habían sido reportados a nivel mundial 23 DDR como modalidad de implementación luego de acuerdos de paz, los cuales se adaptan y contextualizan de acuerdo con cada situación nacional específica de donde se implementen, pero de manera general, estos procesos recogen lecciones aprendidas que posibilitan ser formulados desde enfoques multidimensionales y flexibles con las 
comunidades intervenidas, que permite más énfasis en la atención integral a la reintegración, que en el desarme. Lo cual favorece que los DDR se estén diseñando con base en el marco constitucional y la perspectiva de Derechos Humanos.

Así, un DDR es viable dependiendo del marco legal, la institucionalidad que lo soporta, los recursos estatales, la confianza entre las partes, las garantías y seguridad comunitaria y humana, el coste según el país, su nivel de vida y la renta por habitante; también, de los esfuerzos puestos al excombatiente, en lo referente a su atención psicosocial y la generación de oportunidades culturales, económicas, educativas, laborales y políticas (Fisas, 2011).

Naciones Unidas en 2010, proporciona una guía operacional llamada "Manual de gestión de programas DDR", en esta guía define elementos de seguimiento y evaluación de un DDR, los procedimientos para elaboración de indicadores, plan de seguimiento y gestión de los riesgos de los programas de DDR y la documentación de las lecciones aprendidas. Dicha guía busca entregar un protocolo estándar para cualquier país, donde la ONU participe como mediador, en sus procesos de paz, con el objetivo de implementar de manera sistemática, controlada y sostenida programas de DDR resultados de acuerdos de paz (ONU, 2010).

El Consejo Nacional de Política Económica y Social República de Colombia (Conpes 3554, 2008) emitió la Política Nacional de Reintegración Social y Económica -PRSE, considerada como un Plan de Estado y de Sociedad que busca promover la incorporación efectiva del desmovilizado con voluntad de paz y de su familia a las redes sociales del Estado y a las comunidades receptoras. Esta Política pretende asegurar la superación de su condición a través de: a) la integración de la oferta social y económica del Estado; b) el acompańamiento para incrementar la probabilidad de que las intervenciones mejoren las condiciones de calidad de vida de la población desmovilizada y de sus familias; y c) la construcción de un marco de corresponsabilidad que por un lado, apoye al desmovilizado a regresar y convivir constructivamente en su entorno familiar y comunitario, y por el otro, lo comprometa a él y a sus dependientes con la superación de su situación y la permanencia en la legalidad.

Para esto, es necesario ejecutar medidas que permitan la inserción y permanencia en la vida legal de la población desmovilizada, mediante la seguridad social y el desarrollo socioeconómico sostenible del país, y de esta forma, consolidar realmente la paz.

\section{El impacto en la sociedad civil de los procesos de Paz}

Los procesos de DDR alrededor del mundo, muestran que la reintegración social, tiene dos enfoques, uno basado en lo personal y otro en comunidades. El personal se define por el desarrollo de estrategias aplicadas de manera individual, como una propuesta de proyecto de vida, que contempla la promoción y desarrollo de habilidades, capacidades $\mathrm{y}$ aptitudes formativas, laborales y ciudadanas necesarias para la interacción hacia una cultura de paz y convivencia social. Este enfoque se ha utilizado más frecuentemente, debido a que puede ser menos complejo de implementar y exige menos recursos que una intervención colectiva (Consejo Nacional de Política Económica y Social República de Colombia, Documento Conpes 3554, 2008).

Elenfoquecomunitario, seplanteadesdela necesidad de construir espacios de interacción efectiva entre las comunidades receptoras y los excombatientes, que permitan su reincorporación social como ciudadanos; requiere que se implementen estrategias de convivencia, construcción de ciudadanía, mediación y reconocimiento, más una efectiva reactivación socioeconómica de todas las comunidades afectadas por el conflicto armado. 
Este enfoque tiene el beneficio adicional de permitir una integración más profunda con políticas de desarrollo, consolidación de paz y reparación integral de las victimas (Ugarriza, 2009).

En esta línea, es importante fortalecer el concepto de ciudadanía, que se puso en vigencia con la declaración universal de los Derechos Humanos (ONU, 1948), que permite que toda persona o colectivo humano, más en un proceso de reintegración por conflicto armado, le apuesten a la construcción como ciudadanos con derechos, del ejercicio de sus facultades políticas y socioculturales de una forma digna como parte de un territorio.

En el mismo sentido, los procesos psicosociales en las comunidades, tienen que ser parte de la reflexión y la propuesta de cambio en contextos de posconflicto, instaurándose como aporte en los procesos políticos que se anclan a la realidad del país, para posibilitar el desarrollo social y humano.

Así, un país cuando toma la decisión de salir del conflicto, tiene que generar acciones de transformación social, que impacte a todos sus habitantes, independiente de su posición ante el conflicto y no únicamente, a quienes de forma directa estuvieron en la confrontación (Turriago y Bustamante, 2003).

Desde esta perspectiva, es necesario abordar conceptos como corresponsabilidad, que apunta a una responsabilidad compartida en lo que tiene que ver con los efectos del conflicto. Hoyos (2002), afirma que la corresponsabilidad, hace referencia a la concurrencia de actores y acciones, encaminadas a la garantía de los Derechos, función que tiene que ser asumida por la sociedad y su gobierno, como partes activas de un Estado; lo cual, permite empoderar a la población civil, para participar de manera activa en la construcción de paz y poderse realmente beneficiar de un posconflicto.

De esta forma, la sociedad civil no sería sólo receptora de los efectos del conflicto, sino, actores activos de resistencia contra la violencia, mediante estrategias construidas de forma participativa y consensuada permitiendo así una continua resignificación individual y colectiva.

La ciudadanía se enmarca en la perspectiva de Derecho, como el eje que permite vivir en sociedad, sobre todo cuando una sociedad tiene aspiraciones de ser pacífica y democrática, equitativa e incluyente. Esto exige retos del orden de la diferencia de intereses en la convivencia, de la búsqueda de consensos a favor del bien común, para poder de forma colectiva construir sociedad en la diversidad (Chaux, Lleras y Velásquez, 2004).

Son los derechos políticos y sociales consagrados en la Constitución Política, los que otorgan a los ciudadanos el estatus civil, que concede un Estado (Corte Constitucional de Colombia, 2015).

Desde esta concepción puede relacionarse también, el concepto de competencias ciudadanas, partiendo de la premisa de que son los conocimientos y habilidades que permiten que un ciudadano actúe de manera constructiva en una sociedad democrática. Estas competencias se evidencian en el diario vivir, en lo que las personas hacen. El ejercicio y prácticas de éstas, es el objetivo fundamental de la formación ciudadana. Sin embargo, se requiere el dominio sobre ciertos conocimientos y estar en un ambiente que favorezca la puesta en práctica de estas competencias. (Ministerio de Educación Nacional de Colombia, 2004, p. 149).

Las competencias ciudadanas como ejercicio de los derechos brindan herramientas básicas para que cada persona pueda respetar, defender y promover su ciudadanía, en las situaciones de su vida cotidiana, en que sus derechos pudieran llegar a ser vulnerados (Ministerio de Educación Nacional de Colombia, 2004). 
Las competencias ciudadanas cobran valor en el contexto del actual proceso de paz en Colombia, necesarias para el cumplimiento de los derechos fundamentales de las personas que están reintegrándose, desde el principio de la verdad, reconciliación y perdón de la población civil (Villarraga, 2015).

Este tendría que ser el eje conductor que de manera compartida, permitiría asumir la responsabilidad y el compromiso como país, de salir del círculo de la guerra. En esta medida los procesos de paz realmente pueden generar un impacto positivo en la sociedad, si se usan como recurso para fortalecer el desarrollo, el bienestar psicológico de los ciudadanos víctimas directas del conflicto armado, pero también las indirectas, que en el caso de Colombia, son todos los conciudadanos.

\section{El Bienestar psicológico como categoría hacia el Desarrollo social}

La perspectiva que se viene planteando en este texto, hace posible analizar el rol activo de los ciudadanos en el desarrollo social, donde la acción tripartita entre las comunidades, el gobierno y sus instituciones, permite que se geste un desarrollo, que trascienda los enfoques tradicionales centrados en el crecimiento económico, la distribución de los bienes y la satisfacción de las necesidades humanas. Es necesario entonces, fortalecer el capital humano, es decir la ciudadanía, que es donde está la clave para el fortalecimiento nacional, como la impulsora de su propia libertad y la responsable de su crecimiento integral (Sen, 2000).

Como se viene exponiendo, esta mirada, implica de forma directa a las personas, como actores principales de la transformación social, lo que impacta necesariamente su bienestar psicológico.

Si se entiende el cambio social de un país desde la formación ciudadana, esto exige prestar importancia a los componentes que cada persona requiere fortalecer para aportar a su contexto sociopolítico.

En este orden, Chaux, et al. (2004), proponen que para la promoción y difusión de las competencias ciudadanas, como habilidades que permiten el ejercicio de la ciudadanía, es fundamental reconocer su clasificación, desde los parámetros del bienestar psicológico, en competencias de conocimiento, las cuales, clasifica como: emocionales, cognitivas e integradoras.

Así, las competencias emocionales se refieren a la habilidad subjetiva de reconocer y responder constructivamente ante las emociones propias y las de los demás. Por ejemplo, poder expresar empatía ante las situaciones de otros, tener capacidad para mirarse a sí mismo y reflexionar sobre lo que se hace, piensa o siente.

Lascompetencia cognitivas, asu vez, son capacidades mentales, como saber llegar a acuerdos de beneficio mutuo e interactuar pacífica y constructivamente, poder evaluar adecuadamente los propósitos en las acciones de los demás, asumir diferentes opciones creativas para resolver un conflicto, considerar los efectos que pueda tener una decisión común y por último, el pensamiento crítico para cuestionar la validez de cualquier creencia, afirmación o fuente de información.

Asimismo, las competencias comunicativas son destrezas para establecer un diálogo constructivo, como por ejemplo, saber escuchar, desarrollar la asertividad y la argumentación en el discurso para expresar y sustentar una posición de manera que los demás puedan comprenderla y evaluarla de manera adecuada.

Así las cosas, las competencias de conocimiento son toda la información que se debe saber y comprender como por ejemplo, conocer los Derechos Humanos, la Constitución Política, las formas y mecanismos de participación política; acciones estas del ejercicio público, que exigen de 
la integración de todas las competencias anteriores, por lo que proponen estos autores, que todas estas competencias deben integrarse-denominándolas, competencias integradoras, que son la articulación de dichas competencias, en una respuesta personal en la vida práctica.

En relación, es necesario definir el concepto de bienestar psicológico, a la luz de los beneficios subjetivos y sociales que trae consigo. Arrogante (2014), cita a Ryan y Deci (2001), para tratar el bienestar psicológico, como un estado subjetivo que se encuentra en cada persona, a partir del desarrollo de sus habilidades y talentos, poniéndolos al servicio del bienestar propio y de los demás. Es decir, estos autores proponen el constructo de bienestar psicológico, centrado en el crecimiento personal, mediante el desarrollo de capacidades y potencialidades. Desde esta perspectiva se considera que una persona está bien psicológicamente cuando desarrolla su verdadero potencial.

El bienestar psicológico es sustentado según, Marsollier y Aparicio (2011), por Veenhoven (1991), como el grado de favorabilidad con que una persona juzga de manera general su vida, en donde su autoevaluación, depende de la integración de los estados emocionales, positivos o negativos $\mathrm{y}$, del componente cognitivo, como procesamiento de la información que permite valorar la satisfacción y realización personal o, la sensación de fracaso y frustración, al comparar las aspiraciones de la persona con los logros obtenidos.

Diener (1994) resalta el componente cognitivo, diferenciándolo de los estados emocionales, en tanto es más invariable, al afirmar que se mantiene más en el tiempo y es el resultado del balance que hace la persona, en función de la información que posee sobre sus propios logros y frustraciones.

El bienestar psicológico entonces puede pensarse como un constructo triárquico, con base en la relación, entre las emociones y la cognición, que hacen posible la integración de las representaciones mentales, generando una percepción de autoeficacia que entra en sintonía con el bienestar psicológico subjetivo (Arrogante, 2014).

En esta vía integradora, Ballesteros, Medina y Caycedo (2006), retoman los desarrollos teóricos sobre el bienestar psicológico de Carol Ryff, quien en la última década del $S$. xx, propuso un modelo multidimensional, el cual como proceso, forma el desarrollo personal, en tanto capacidad subjetiva de asumir compromiso con los retos existenciales de la vida. Este desarrollo es propuesto como un continuo a través del ciclo vital, que permite fortalecer el potencial de cada persona.

Es así que el Bienestar psicológico se fortalece a partir de una transacción entre las características del individuo y las circunstancias y posibilidades que le ofrece su entorno micro y macro social (García y González, 2000), dicha transacción involucra un ajuste a nivel subjetivo, de aspectos vigentes e históricos, que contienen recursos y condiciones materiales, emocionales, cognitivas, que propician el sentido de satisfacción con la vida, que es en última instancia lo que se percibe como condición para la realización personal (Ciarrochi y West, 2004).

Por tanto, las concepciones sobre el bienestar psicológico provienen de modelos del desarrollo psicológico y del ciclo vital, que ponen de manifiesto teorías motivacionales, desde categorías personales y dimensiones contextuales de la vida cotidiana de las personas, que apuntan a la calidad de vida, a la salud mental (Bradburn, 1969) y (Vaillant, 2000).

El enfoque multidimensional del Bienestar psicológico de Ryff (1989) plantea un proceso de desarrollo personal que implica la salud integral, que trasciende lo biológico, a la estabilidad emocional, a la calidad en los niveles de interacción familiar y social, a la autorrealización en el trabajo y proyecto de vida. Estas cualidades psicosociales conformarían el bienestar psicológico, manifestándose en todas las esferas de la actividad humana (Flórez-Alarcón, 2006). 
En el contexto del posconflicto y el proceso de paz en Colombia, es fundamental tener presente este enfoque al momento de hacer propuestas integradoras de reintegración social.

De tal forma, que analizar el bienestar psicológico de una persona que se ha desvinculado del conflicto armado, exige pensar asuntos del orden de la percepción que tiene de sí mismo, sus creencias, sus formas de vinculación familiar y en su entorno social inmediato, su formación académica y laboral, la manera cómo afronta sus retos cotidianos, todo esto para poder valorar, en términos de bienestar psicológico, el esfuerzo que hace para alcanzar sus metas y proyecciones de vida, desde la auto aceptación de la vida pasada.

Pues como plantean Ryffy Keyes (1995), el bienestar psicológico es un continuo, que según el momento del ciclo vital, va fortaleciéndose en beneficio de proyecciones personales hacia el contexto, lo cual va generando ganancias en la autonomía.

Asimismo, el bienestar psicológico, necesariamente comprende el valor que se le otorga a las circunstancias cotidianas dentro del contexto social, como una forma de satisfacción personal, que surge como beneplácito entre la persona y su contexto, el cual implica, las dimensiones culturales e históricas, que a su vez incluyen condiciones objetivas materiales y psicosociales, que brindan determinadas oportunidades para la realización personal (García y González, 2000).

De esta manera, el Bienestar psicológico, puede definirse en términos de desarrollo humano, como un factor principal de la calidad de la vida integral de las personas a nivel subjetivo y colectivo.

\section{El desarrollo humano en el posconflicto}

Valcárcel (2006) plantea que el desarrollo tiene que entenderse como la capacidad de una comunidad de consolidar sus lazos sociales al interior de una base territorial definida, que les permita cohesionar una identidad cultural, en el marco de un soporte institucional que garantice sus derechos.

En esta medida el desarrollo estará enriquecido por la diversidad, que hace posible la trasformación de las realidades sociales, cuando el desarrollo se enfoca en lo humano (Informe del Desarrollo Humano, 1990).

En un contexto de posconflicto, implementando medidas de reintegración social, comprender el desarrollo, desde una mirada enfocada en lo humano, hace posible que se asuma como un proceso mediante el cual, se amplían las potencialidades y oportunidades de las personas, entre las cuales, las más importantes son, una vida prolongada y saludable, el acceso a la educación, al trabajo digno, el disfrute de un nivel de vida decente. Otras oportunidades incluyen la libertad política, la garantía de los derechos humanos y el respeto a sí mismo (PNUD, 2010).

Sen (2000) aporta a este concepto desde una perspectiva de distribución de los bienes, la satisfacción de las necesidades humanas y la equidad, a partir de la concepción de libertades, capacidades y agencia; fundando una idea del desarrollo humano, en el aumento de la riqueza de la vida humana en lugar de la riqueza económica.

La relación entre desarrollo humano y la libertad subjetiva, va más allá de las oportunidades económicas y políticas, es decir, lo que cada persona puede conseguir depende de dichas oportunidades, pero también de las iniciativas y cohesiones sociales y de la libertad para ser partícipe de las decisiones públicas (García Peña, 2015).

Esto significa que cada persona, dentro de su colectivo comunitario, es responsable de su desarrollo, lo cual, hace que sea un agente activo del desarrollo, con capacidad de autogestión en relación con su contexto social. De ahí la importancia, que en un momento histórico como un posconflicto, sea la oportunidad, para que las 
personas, aprendan a conocerse en su dimensión psicosocial, moviéndose del lugar de actores pasivos del sistema, que los ubica como objetos de las condiciones y ayudas externas. Así, se otorga al ser humano la facultad de actuar y generar los cambios para encontrar el desarrollo humano, integral y sostenible de acuerdo con las realidades e intereses que se tengan (García Peña, 2015).

En esta misma línea, el Desarrollo humano es visto como una capacidad de las personas en pro de su comunidad o territorio, el cual, se da como un proceso de participación activa, partiendo de su dinámica particular y de sus propios recursos personales (bienestar psicológico), para potencializar acciones corresponsables que conduzcan al progreso del colectivo. En síntesis, todo proceso de cambio, como lo exige un proceso de reintegración social, debe apuntar al desarrollo, más allá de la dinámica política que lo soporte y de su rentabilidad económica.

Con este recorrido teórico, desde una visión integradora de las personas como eje central del desarrollo social, pasando por los procesos de reintegración, hasta llegar a la perspectiva de los derechos y el ejercicio de ciudadanía que atañe a cada persona, hace que se piense lo psicosocial como un proceso de desarrollo de los contextos, que puede verse reflejado en los estados de bienestar de las personas en un colectivo.

\section{Conclusiones}

Es necesario reconocer la función que cumple la sociedad civil en la aprobación de los procesos de reintegración social, pues es en la sociedad, donde se comparten los espacios de integración en el posconflicto, lo cual genera un impacto en las dinámicas culturales, políticas y económicas del país.

Por ello se requiere, en aras de la reintegración social, fortalecer los procesos de perdón $y$ reconciliación, para hacer posible la convivencia y la transformación a mediano plazo, de las concepciones que la sociedad tiene del conflicto, la institucionalidad y el desarrollo del país.

Por esto es parte del posconflicto reconocer a las personas que han dejado las armas como parte de la sociedad, como sujetos de derecho, con posibilidades de acceso a su ciudadanía, protegidos por la constitución política, con derecho a educación, salud, vivienda, trabajo digno y ejercicio pleno de su civilidad. Esta será la única forma de borrar el estigma de su vida pasada y que se dé realmente la reintegración social, donde el resto de la sociedad logre dejar la desconfianza y el temor.

Estigmatizar y rechazar a las personas en proceso de reintegración social, problematiza a la sociedad misma, con su propósito de alcanzar una verdadera paz, al desconocer el conflicto como parte del desarrollo humano; esto sugiere, que las estrategias que se implementen en el ejercicio de la reintegración, sean del conocimiento de la ciudadanía, como una pedagogía en competencias ciudadanas, tanto a nivel del sector educativo, los medios de comunicación, las instituciones públicas y el sector privado; de lo contrario, el proceso de paz, queda supeditado sólo a la voluntad de una élite y no al consenso de todo el país.

Es importante que la sociedad civil esté informada y formada, y entre estos que, la más afectada por el conflicto, asuma un papel protagónico, activo, de formación ciudadana e incorporación pedagógica de la paz, mediante encuentros sociales, foros temáticos, redes sociales, desde iniciativas propias y apoyadas por el gobierno.

De esa forma se podría pensar como resultado del posconflicto, un país más justo, equitativo, en pro de la igualdad de oportunidades de sus ciudadanos.

En un proceso de posconflicto es obligatorio reconocer objetivamente las necesidades emocionales, cognitivas y psicosociales de las 
personas que se encuentren en proceso de reintegración social por haber pertenecido al conflicto armado, porque de esta forma se contribuye a identificar las condiciones personales de esta población, para así poder, comprender las circunstancias que les llevaron al conflicto, igualmente las situaciones de contexto de nuestro territorio y las formas que permitirán hacer más efectiva la reintegración social, como un proceso equitativo de todo el país, donde no se puede seguir mirando de forma excluyente a sectores de la población. Solo así, podría ser exitoso este proceso de construcción de paz.

Las experiencias de reintegración social en Colombia se han quedado cortas, porque se han supeditado a la aplicación de estrategias que proporcionan herramientas prácticas a las personas desvinculadas del conflicto, de tal manera que a partir del aprendizaje de un oficio puedan acceder a su sustento; por otro lado, sería más conveniente, que para estos procesos tan complejos, las acciones de intervención fueran pertinentes, generando estrategias de formación, que reconozcan las condiciones personales de esta población, siendo estas el insumo para realizar acciones efectivas de reintegración familiar, educativa, laboral y social, en las cuales además, se incluya, no solo a los excombatientes, si no la sociedad en general.

Se requiere un enfoque de atención integral de larga duración para los procesos de reintegración social de las personas que se han desvinculado del conflicto armado. Lo cual exige, por parte del Estado, realizar estrategias concretas que hagan posible un desarrollo de las personas, en su bienestar psicológico, mediante un fortalecimiento emocional, cognitivo y social que les permita superar su historia de vulnerabilidad y les prepare para el proceso de reintegración que ofrece el posconflicto.

Todo este esfuerzo por construir la paz debe incluir la participación de agentes de cambio comprometidos, donde todos los sectores estatales generen espacios de inclusión que vayan más allá de las ayudas económicas y empoderen a cada persona desvinculada del conflicto de su desarrollo, brindando las herramientas, que permitan potenciar su actuación como ciudadano.

Por último, estas reflexiones también pueden permitir nuevos espacios investigativos enfocados a la intervención de la salud mental de las personas desvinculadas del conflicto armado y que en este momento histórico del país, hacen parte activa del contexto social y cultural.

\section{Referencias}

ACR-Agencia colombiana para la Reintegración (2016). Resolución 1356. "Por la cual se modifican los artículos 3,4,17,19,21,26,31,32,37,39,44 y 46 y se deroga el artículo 38 de la resolución 0754 de 2013”. Recuperado de http://www.reintegracion. gov.co/es/agencia/Documentos\%20Resoluciones/ Resoluci\%C3\%B3n\%201356.pdf

Agencia colombiana para la Reintegración de personas y grupos alzados en armas (2017). Recuperado de http://www.reintegracion.gov.co/es

Agencia para la Reincorporación y la Normalización - ARN (2017). Informe de resultados, Audiencia pública de rendición de cuentas a la ciudadanía de la Agencia colombiana para la reintegración de personas y grupos alzados en armas - ACR, vigencia 2016. Recuperado de: http://www.reintegracion.gov.co/ es/agencia/Documentos\%20de\%20informes $\% 20$ de $\% 20$ rendicin $\% 20$ de $\% 20$ cuentas/Informe $\% 20$ Rendici\%C3\%B3n\%20de\%20Cuentas\%20 Final\%202017.pdf

Arrogante, Ó. (2014). Estudio del bienestar en personal sanitario: relaciones con resiliencia, apoyo social, estrés laboral y afrontamiento (Tesis doctoral). UNED. Recuperado de: https://dialnet.unirioja. es/servlet/tesis?codigo $=44107$.

Ballesteros, B., Medina, A., y Caycedo, C. (2006). El bienestar psicológico definido por 
asistentes a un servicio de consulta psicológica en Bogotá, Colombia. Universitas psychologica, 5(2), 239-258. Recuperado de http://www.scielo.org.co/scielo.php?script $=$ sci_arttext \&pi$\mathrm{d}=$ S1657-92672006000200004

Bradburn N.M. (1969). The structure of psychological well-being. Chicago: Aldine.

Centro Nacional de Memoria Histórica - CNMH (2015). Desmovilización y reintegración paramilitar. Panorama posacuerdos con las AUC. Recuperado de: http://www.centrodememoriahistorica.gov.co/descargas/informes2015/desmovilizacionDesarmeReintegracion/desmovilizacion-y-reintegracion-paramilitar.pdf

Chaux, E., Llenas, J. y Velásquez, A. (2004). Competencias Ciudadanas: De los Estándares al Aula Una propuesta de integración a las áreas académicas. Ministerio de Educación Nacional de Colombia. Recuperado de http://www.colombiaaprende.edu.co/ html/mediateca/1607/articles-75077_archivo.pdf

Ciarrochi, J. y West, M. (2004). Relationships between dysfunctional beliefs and positive and negative indices of well-being: A critical evaluation of the common beliefs survey-III. Journal of RationalEmotive and Cognitive Behavior Therapy. 22 (3). 171-188.

Consejo Nacional de Política Económica y Social República de Colombia Departamento Nacional de Planeación (2008). Documento Conpes 3554. Politica Nacional de reintegración social y económica para personas y grupos armados ilegales. Alta consejería para la reintegración. Bogotá, Colombia. Recuperado de: https://www.reintegracion.gov. co/es/la-reintegracion/centro-de-documentacion/Documentos/Documento\%20Conpes $\% 20$ 3554\%201\%20Pol\%C3\%ADtica $\% 20$ nacional $\% 20$ de $\% 20$ reintegraci $\%$ C3 $\%$ B 3 n $\% 20$ social $\% 20 y \% 20$ econ $\%$ C3\%B 3 mica $\% 20$ para $\% 20$ personas $\% 20 \mathrm{y} \% 20$ grupos $\% 20$ armados $\% 20$ ilegales.pdf
Corte Constitucional de Colombia, 2015. Constitución Política de Colombia y actualización con los Actos Legislativos a 2015. Recuperado de http://www.corteconstitucional.gov.co/inicio /Constitucion \%20politica\%20de\%20Colom bia\%20-\%202015.pdf

Diener, E. (1994). Assessing subjective well-being: Progress and opportunities. Social indicators research, 31(2), 103-157. Recuperado de https://link. springer.com/article/10.1007\%2FBF01207052

Fisas, Vicenç (2010). Procesos de paz comparados. Cuaderno No. 14 de los Quaderns de Construcción de Pau de la ACCD (Agencia Catalana de Cooperació al Desenvolupament)- ECP (Escola de Cultura de Pau). Recuperado de http://escolapau. uab.es/img/qcp/procesos_paz_comparados.pdf

Fisas, Vicenç (2011). Introducción al Desarme, Desmovilización y Reintegración (DDR) de excombatientes. Cuaderno No. 24 de los Quaderns de Construcción de Pau de la ACCD (Agencia Catalana de Cooperació al Desenvolupament)ECP (Escola de Cultura de Pau). Recuperado de http://escolapau.uab.es/img/qcp/introduccion_ ddr.pdf

Flórez-Alarcón, L. (2006). La psicología de la salud en Colombia. Universitas psychologica. 5 (3). 681-693.

García, C. González, I. (2000). La categoría bienestar psicológico: Su relación con otras categorías sociales. Revista cubana de medicina general integral. 16 (6). 586-592.

García Peña, J. J. (2015). Intervención psicosocial como aporte al desarrollo humano local: en el ámbito público de Medellin. Saarbrucken, Alemania: Editorial Académica Espańola.

GMH. (2013). ¡Basta Ya! Colombia: Memorias de guerra y dignidad. Grupo de Memoria Histórica. Santa Fé de Bogotá. Imprenta Nacional. 
Hoyos, C. (2012). La corresponsabilidad familiar: Rupturas históricas y prospectiva en Medellin. Colombia: Unaula.

Informe del desarrollo humano (1990). PNUD. Tercer mundo editores. Oxford University Press. Recuperado de http:/hdr.undp.org/sites/default/ files/hdr_1990_es_completo_nostats.pdf

Marsollier, R., y Aparicio, M. (2011). El bienestar psicológico en el trabajo y su vinculación con el afrontamiento en situaciones conflictivas. Psicoperspectivas, 10(1), 209-220. Recuperado de http://www.scielo.cl/scielo.php?pi$\mathrm{d}=$ S0718-69242011000100011\&script=sci_arttext.

Ministerio de Educación Nacional de Colombia (2004). Formar para la ciudadanía. Serie de cartillas No 6: Estándares Básicos de Competencias Ciudadanas. Lo que necesitamos saber y saber hacer. Recuperado de: http://www.mineducacion. gov.co/cvn/1665/articles-75768_archivo_pdf.pdf

Organización de las Naciones Unidas ONU. (1948). Declaración Universal de los Derechos Humanos. Asamblea General de las Naciones Unidas, Resolución 217 A (III), París. Recuperado de http://www.un.org/es/ universal-declaration-human-rights/

PNUD - Programa de las Naciones Unidas para el Desarrollo. (2010.). Informe de Desarrollo Humano, La verdadera riqueza de las naciones: Caminos al desarrollo humano. Edición aniversario. USA.

Presidencia de la República. (2011). Decreto N. 4138 de 2011 "Por el cual se crea la Agencia Colombiana para la Reintegración de Personas y Grupos Alzados en Armas y se establecen sus objetivos y estructura". Recuperado de http://www.reintegracion.gov.co/es/agencia/Documentos\%20Decretos/ Forms/DispForm.aspx?ID=4

Presidencia de la República. (2015). Decreto $N$. 2253 de 2015 "Por el cual se modifica la estructura de la Agencia Colombiana para la Reintegración de
Personas y Grupos Alzados en Armas". Recuperado de http://www.reintegracion.gov.co/es/agencia/ Documentos\%20Decretos/Decreto\%202253\%20 de\%202015.pdf

Presidencia de la República (2017). Decreto $N$. 897 de 2017 "Por el cual se modifica la estructura de la Agencia Colombiana para la Reintegración de Personas y Grupos Alzados en Armas y se dictan otras disposiciones". Recuperado de http://es.presidencia.gov.co/normativa/normativa/DECRETO $\% 20$ 897\%20DEL\%2029\%20DE\%20MAYO\%20 DE\%202017.pdf.

ONU (2010). Second Generation Disarmament, Demobilization and Reintegration (DDR) Practices in Peace Operations. A New Contribution to the New Horizon Discussion on Challenges and Opportunities for UN Peacekeeping. Nueva York: Departamento de Operaciones de Paz. Naciones Unidas.

Ryff, C. D. (1989). Happiness is everything, or is it? Explorations on the meaning of psychological well-being. Journal of Personality and Social Psycholog. 6 (57). 1069-1089. University of Wisconsin-Madison. Recuperado de http://www.docslides.com/sherrill-nordquist/ journal-o-personalit-an-socia

Ryff, C. D., Keyes. L.M. (1995). The Structure of Psychological Well-Being Revisited. Journal of Personality and Social Psychology. 69 (4). 719-727. University of Wisconsin-Madison. Recuperado de http://midus.wisc.edu/findings/pdfs/830.pdf

Sen, A. (2000). Desarrollo y libertad. Barcelona: Ed. Planeta S.A.

Turriago, G. y Bustamante, J.M. (2003). Estudio de los procesos de reinserción para un análisis del posconficto en Colombia -1991-1998-. Fundación Ideas para la Paz. Recuperado de http://www.ideaspaz. org/publications

Ugarriza, J. y Mesías L. (2009). Dilemas de la reintegración de ex-combatientes en Bogotá. Preparado 
para el VIII seminario de investigación urbano-regional, universidad nacional, Bogotá, septiembre 28-30. Recuperado de http://centrodememoriahistorica.gov.co/descargas/informes2012/encuesta. pdf

Vaillant, George E. (2000). Adaptive Mental Mechanisms: Their Role in a Positive Psychology. American Psychologist. 55(1), 89-98. Boston - MA. Valcárcel, M. (2006). Génesis y evolución del concepto y enfoques sobre el desarrollo (Documento de investigación). Departamento de Ciencias
Sociales. Pontificia Universidad Católica del Perú. Recuperado de http://www.ucipfg.com/ Repositorio/MGTS/MGTS15/MGTSV15-01/ SEMANA1/71583949-Genesis-y-Evolucion-DelConcepto-de-Desarrollo.pdf

Villarraga, Álvaro. (2015). Desmovilización y reintegración paramilitar panorama posacuerdos con las AUC. Bogotá, Centro Nacional de Memoria Histórica. Recuperado de http://babel.banrepcultural.org/cdm/ref/collection/p17054coll2/id/52

\section{Datos de filiación}

John Jairo García Peña. Magíster en Desarrollo, Universidad Pontificia Bolivariana; especialista en psicoanálisis con niños, Universidad de Antioquia; psicólogo egresado de Antioquia. Docente e investigador escuela de posgrados Universidad Católica Luis Amigó. Correo-e: john.garciape@amigo.edu

Claudia Marcela Arana Medina. Psicóloga, Universidad San Buenaventura; especialista en terapia cognitiva y en psicología organizacional, Universidad San Buenaventura; magíster en Neuropsicología, Universidad San Buenaventura y Ph.D. en Psicología con Orientación en Neurociencia Cognitiva Aplicada por la Universidad Maimónides. Docente Universidad de Sanbuenaventura y Universidad Católica Luis Amigó. Correo-e: claudia.araname@amigo.edu.co 